\title{
Effectiveness of rotavirus vaccination in prevention of hospital admissions for rotavirus gastroenteritis among young children in Belgium: case-control study
}

\author{
(c) $(1) \circledast$ Or OPEN ACCESS
}

\section{Tessa Braeckman predoctoral researcher ${ }^{1}$, Koen Van Herck senior lecturer in vaccinology and} public health $^{12}$, Nadia Meyer epidemiology director ${ }^{3}$, Jean-Yves Pirçon study biostatistician ${ }^{3}$, Montse Soriano-Gabarró head of global epidemiology ${ }^{4}$, Elisabeth Heylen predoctoral researcher ${ }^{5}$, Mark Zeller predoctoral researcher ${ }^{5}$, Myriam Azou paediatrician ${ }^{6}$, Heidi Capiau paediatrician ${ }^{7}$, Jan De Koster paediatrician ${ }^{8}$, Anne-Sophie Maernoudt paediatrician ${ }^{9}$, Marc Raes paediatrician ${ }^{10}$, Lutgard Verdonck paediatrician $^{11}$, Marc Verghote paediatrician ${ }^{12}$, Anne Vergison paediatrician ${ }^{13}$, Jelle Matthijnssens postdoctoral researcher ${ }^{5}$, Marc Van Ranst professor faculty of medicine ${ }^{5}$, Pierre Van Damme professor faculty of medicine ${ }^{1}$, on behalf of the RotaBel Study Group

${ }^{1}$ Centre for the Evaluation of Vaccination, Vaccine and Infectious Disease Institute, University of Antwerp, Antwerp, Belgium; ${ }^{2} \mathrm{Public}$ Health Department, Ghent University, Ghent, Belgium; ${ }^{3}$ GlaxoSmithKline Biologicals, Wavre, Belgium; ${ }^{4}$ Bayer Healthcare Pharmaceuticals, Berlin, Germany; ${ }^{5}$ Clinical and Epidemiological Virology, KU Leuven, Belgium; ${ }^{6} \mathrm{AZ}$ Damiaan, Paediatric Department, Ostend, Belgium; ${ }^{7} \mathrm{AZ}$ St Lucas, Paediatric Department, Ghent; ${ }^{8} \mathrm{ZOL}$ (Ziekenhuis Oost-Limburg), Campus Sint-Jan, Department of Paediatrics, Genk, Belgium; ${ }^{9} \mathrm{Clinic}$ St Pierre, Paediatric Department, Ottignies, Belgium; ${ }^{10} \mathrm{Jessa}$ Hospital, Paediatric Department, Hasselt, Belgium; ${ }^{11} \mathrm{AZ}$ Alma, Paediatric Department, Eeklo, Belgium; ${ }^{12} \mathrm{CHR}$ Namur, Paediatric Department, Namur, Belgium; ${ }^{13}$ University Hospital for Children, Infectious Diseases Unit, Brussels

\begin{abstract}
Objective To evaluate the effectiveness of rotavirus vaccination among young children in Belgium.

Design Prospective case-control study.

Setting Random sample of 39 Belgian hospitals, February 2008 to June 2010.

Participants 215 children admitted to hospital with rotavirus gastroenteritis confirmed by polymerase chain reaction and 276 age and hospital matched controls. All children were of an eligible age to have received rotavirus vaccination (that is, born after 1 October 2006 and aged $\geq 14$ weeks).

Main outcome measure Vaccination status of children admitted to hospital with rotavirus gastroenteritis and matched controls.

Results 99 children (48\%) admitted with rotavirus gastroenteritis and $244(91 \%)$ controls had received at least one dose of any rotavirus vaccine $(\mathrm{P}<0.001)$. The monovalent rotavirus vaccine accounted for $92 \%(n=594)$ of all rotavirus vaccine doses. With hospital admission as the outcome, the unadjusted effectiveness of two doses of the
\end{abstract}

monovalent rotavirus vaccine was $90 \%$ (95\% confidence interval $81 \%$ to $95 \%$ ) overall, $91 \%$ (75\% to $97 \%$ ) in children aged $3-11$ months, and $90 \%(76 \%$ to $96 \%)$ in those aged $\geq 12$ months. The G2P[4] genotype accounted for $52 \%$ of cases confirmed by polymerase chain reaction with eligible matched controls. Vaccine effectiveness was $85 \%$ (64\% to $94 \%$ ) against G2P[4] and 95\% (78\% to 99\%) against G1P[8]. In 25\% of cases confirmed by polymerase chain reaction with eligible matched controls, there was reported co-infection with adenovirus, astrovirus and/or norovirus. Vaccine effectiveness against co-infected cases was $86 \%(52 \%$ to $96 \%$ ). Effectiveness of at least one dose of any rotavirus vaccine (intention to vaccinate analysis) was $91 \%$ (82\% to $95 \%$ ).

Conclusions Rotavirus vaccination is effective for the prevention of admission to hospital for rotavirus gastroenteritis among young children in Belgium, despite the high prevalence of G2P[4] and viral co-infection.

\section{Introduction}

Rotavirus is the most common cause of severe acute gastroenteritis in infants and young children worldwide. ${ }^{1}$ Nearly every child will have experienced a symptomatic infection 
before the age of 5 years, ${ }^{12}$ with the peak incidence occurring among children aged 4-23 months. ${ }^{34}$ Although rarely fatal in high income regions, ${ }^{12}$ rotavirus gastroenteritis places a high demand on European healthcare systems. ${ }^{5-8}$ Surveillance studies have shown that rotavirus accounts for up to two thirds of admissions to hospital and emergency room visits and one third of primary care consultations for acute gastroenteritis among children under 5 years in Europe, with the greatest burden of disease consistently seen in children aged under $2 .^{6-8}$ In Belgium, rotavirus gastroenteritis was estimated to account on average for 5674 admissions to hospital (including nosocomial infections) and 26772 ambulatory visits among children aged under 7 from 2000 to 2006 (including visits to general practitioners and paediatricians). ${ }^{9}$

To reduce the burden of rotavirus disease, the World Health Organization recommends inclusion of rotavirus vaccines into all national immunisation programmes. ${ }^{10}$ Two oral rotavirus vaccines are now available worldwide, a monovalent human rotavirus vaccine (Rotarix; GlaxoSmithKline Biologicals, Rixensart, Belgium) and a pentavalent bovine-human reassortant rotavirus vaccine (RotaTeq; Merck, Whitehouse Station, NJ). Both vaccines are highly efficacious for the prevention of rotavirus gastroenteritis in large scale clinical trials. ${ }^{11-17}$ These data suggest that vaccination has the potential to significantly reduce the global burden of rotavirus disease. It is essential, however, to establish the effectiveness of the vaccine under conditions of routine use. The effectiveness of rotavirus vaccine during routine use has been reported mainly in low-middle income settings. ${ }^{18} 19$

Belgium was the first country in the European Union to include rotavirus vaccine in the routine infant vaccination schedule, ${ }^{20}$ with rotavirus vaccination recommended since October 2006 and partially reimbursed since November 2006, resulting in a copayment by the parents of $€ 10$ (about $£ 8$ or $\$ 12$ ) per dose. Uptake in Belgium has been rapid, with coverage rates already over $90 \% .{ }^{20}$ Modelling estimates suggest that a fully funded universal rotavirus vaccination programme in Belgium with uptake rates similar to those for other routine infant vaccinations could reduce the annual number of hospital admissions for rotavirus gastroenteritis by as much as $87 \%{ }^{21}$

We undertook a case-control study to estimate the effectiveness of rotavirus vaccination for the prevention of admission to hospital for rotavirus gastroenteritis among young children in Belgium. We also collected data on the burden of rotavirus disease, distribution of rotavirus genotypes, and co-infections with other common intestinal viruses.

\section{Methods}

\section{Study design}

This was a prospective, hospital based, multicentre, matched case-control study. Hospitals with paediatric beds in Belgium were invited at random (following a list generated by random sampling without replacement with R Statistical software (R Foundation for Statistical Computing, Vienna, 2005)). We contacted 60 hospitals to obtain the anticipated 39 hospitals willing to participate in this study, representing about a third of all hospitals with paediatric beds in Belgium and 1073 of the total 2787 paediatric beds. Reasons for refusal to take part included lack of time, lack of qualified personnel, closure of the paediatric ward, and patient population not suitable for aim of the study. Study design was based on the WHO generic protocol for monitoring the impact of rotavirus vaccination on the burden of gastroenteritis disease. ${ }^{22}$

\section{Participants Cases}

We identified cases of gastroenteritis among children eligible to have received at least one dose of any rotavirus vaccine (that is, aged at least 14 weeks of age and born after 1 October 2006). We reviewed admission logs to identify those with onset within 14 days of admission to hospital to determine eligibility for inclusion in the study. Gastroenteritis was defined as at least two episodes of vomiting or three episodes of diarrhoea, or both, within a 24 hour period that were not because of an underlying medical condition and that required at least one overnight stay with oral or intravenous rehydration (equivalent to WHO plan $\mathrm{B}$ or C). Stool samples were collected from eligible children within 48 hours of admission and tested for the presence of rotavirus with a rapid test (Rotastrip or Combistrip; Coris BioConcept, Wepion, Belgium). Samples with positive results for rotavirus by rapid test were stored at $2-8^{\circ} \mathrm{C}$ and sent to the Laboratory of Clinical and Epidemiological Virology at the University of Leuven for confirmation and genetic characterisation of rotavirus infection by polymerase chain reaction followed by sequencing. Samples confirmed to be positive for rotavirus by polymerase chain reaction were also tested for the presence of other common intestinal viruses (adenovirus, astrovirus, and norovirus).

Children were not considered for inclusion in the study if they had previously participated, if they had nosocomial gastroenteritis, or if they had a condition where rotavirus vaccination was contraindicated (including hypersensitivity to active substance or any of the excipients of the rotavirus vaccines, hypersensitivity after previous administration of rotavirus vaccines, previous history of intussusception, uncorrected congenital malformation of the gastrointestinal tract that would predispose for intussusception, known or suspected immunodeficiency, malignancies, receipt of immunosuppressive treatment).

\section{Controls}

For each child with rotavirus gastroenteritis confirmed by polymerase chain reaction, we identified one at least control child who matched the case by date of birth (up to a maximum of six weeks before or after) and was admitted to or was attending an outpatient clinic at the same hospital for any reason except gastroenteritis during the same time period. Eligible controls were listed according to the date of admission/attending date and participation was requested in chronological order. Children were not considered for inclusion in the study if they had previously participated, if they had symptoms of nosocomial gastroenteritis, or if they had a condition where rotavirus vaccination was contraindicated.

\section{Data collection}

For all children we interviewed parents and reviewed medical records to obtain information on demographics, medical history (including previous admission for gastroenteritis), current feeding practice, socioeconomic status, and the current episode of gastroenteritis (cases only). All reasonable efforts (several phone calls or emails, including at least one letter by registered mail) were made to confirm vaccination history (including the brand of vaccine used, number of doses administered, and dates of vaccination) from written sources-for instance, by vaccination card or review of medical record. 


\section{Sample size for vaccine effectiveness}

Our primary analysis assessed the association between receipt of two doses of monovalent rotavirus vaccine and admissions to hospital for rotavirus gastroenteritis, therefore our precision based sample size calculation was based on following assumptions: rotavirus vaccine coverage rates in Belgium of $90 \%$, with a market share for the monovalent rotavirus vaccine of $80 \%$; expected vaccine effectiveness of $80 \%$; and an annual background incidence rate of rotavirus in Belgium of 5000 admissions for rotavirus gastroenteritis in children aged under 6 years, ${ }^{23}$ with $50 \%$ of cases occurring in children under 1 year and $38 \%$ of cases occurring in children aged 1-2 years. After amendment of the case-control ratio from 2:1 to 1:1 (because of difficulties in finding controls), we estimated that we needed 222 children admitted with rotavirus gastroenteritis confirmed by polymerase chain reaction (and 222 age and hospital matched controls) to provide $90 \%$ power, as initially planned, to show the effectiveness of full series monovalent rotavirus vaccine with a threshold of the lower limit of the two sided $95 \%$ confidence interval equal to $50 \%$. In addition, we assumed that we would need to exclude $15 \%$ of confirmed cases from the analysis (for example, because of the absence of age matched controls), that $10 \%$ of children testing positive for rotavirus with the rapid test would test negative by polymerase chain reaction, and that rotavirus is responsible for about half of all cases of gastroenteritis in the study population. We therefore aimed to enrol 560 children with gastroenteritis.

\section{Statistical analysis}

Our primary objective was to estimate the effectiveness of the full two dose course of the monovalent rotavirus vaccine for the prevention of rotavirus gastroenteritis confirmed by polymerase chain reaction and requiring admission to hospital among age eligible children born after 1 October 2006 and aged at least 14 weeks. The primary analysis of effectiveness included only pairs in which the affected child (case) and the control had received either two doses of the monovalent rotavirus vaccine or no rotavirus vaccine at all and who met all criteria defined in the protocol. When we derived the vaccination status for the case and matched control(s), we considered only vaccine doses administered at least 14 days before the onset date of gastroenteritis.

We estimated vaccine effectiveness (\%) as (1-matched odds ratio of vaccination) $\times 100$. The matched odds ratio for vaccination was calculated as a hazard ratio by using conditional logistic regression with $95 \%$ confidence intervals. To identify variables that could affect the estimate, we used models controlling for factors potentially associated with vaccination and rotavirus disease, including sex, attendance at day care, attendance at preschool, medical history, history of breast feeding, maternal education level, and household size. We selected significant factors with a backward strategy, with $\mathrm{P}<0.20$ leading to retention in the model. Vaccine effectiveness of the full two dose course of the monovalent rotavirus vaccine was also estimated according to age at onset of disease (3-11 months and $\geq 12$ months; for controls, age was computed at the date of onset of disease of the matched case), severity of rotavirus gastroenteritis determined with the Vesikari scale (calculated with data available up to the visit and not for the full duration of the episode of gastroenteritis), ${ }^{24}$ rotavirus genotype, and the presence of common viral intestinal co-infections. A Vesikari score of 1-10 was considered to indicate mild or moderate disease, while a score of 11 or greater was indicated severe disease (see appendix). ${ }^{24} \mathrm{We}$ also estimated the effectiveness of at least one dose of any rotavirus vaccine (intention to vaccinate analysis). For all estimates of vaccine effectiveness, we performed a sensitivity analysis, assuming that cases and controls with missing or unknown history of vaccination were, respectively, vaccinated and unvaccinated (sensitivity -), or vice versa (sensitivity + ). Demographic characteristics of cases and the controls were compared with Fisher's exact test for categorical variables and Student's $t$ test for continuous variables. $\mathrm{P}<0.05$ was considered significant.

As a secondary objective, we calculated the proportion of admissions for gastroenteritis and the proportion of admissions attributable to rotavirus infection among age eligible children with exact $95 \%$ confidence intervals.

All statistical analyses were performed with SAS statistical software (version 9.1, SAS, Cary, NC).

\section{Results \\ Study population}

Between February 2008 and June 2010, a total of 4742 age eligible children admitted for gastroenteritis were screened for inclusion in the study (fig $1 \Downarrow$ ). We enrolled 554 children with gastroenteritis (cases) and 352 controls. Of these, 215 cases and 276 controls were eligible for inclusion in the ATP (according to protocol) confirmed cohort for analysis of vaccine effectiveness (61 cases had two matched controls). Of the 276 controls, $53 \%(n=147)$ were admitted to hospital. The absolute median difference between date of birth in cases and matched controls was two weeks (range zero to six weeks). The absolute median time difference between the date of admission in cases and the admission/attending date of matched controls was five weeks (range zero to 100 weeks).

Table 1 shows the demographic characteristics of the "according to protocol" confirmed cohort (cases and controls) $\downarrow$. Median age at enrolment was 12 months (range 3-31 months) for cases and 15 months (3-39 months) for controls. This apparent difference was caused by a time lag in the enrolment of controls. The age of cases and controls at the onset of disease of the matched case, however, was similar, indicating that the age matching was successful (table 1). No significant differences were seen between cases and controls in terms of previous admission for gastroenteritis, medical history, or attendance at day care (table $2 \Downarrow$ ). Compared with controls, however, in cases children were more commonly formula fed, came from a larger size household, had mothers with a lower education level (proxy for socioeconomic status), and were less likely to attend preschool. Concerning current feeding practice, only $4 \%$ of controls and $2 \%$ of cases were breast fed and differences regarding formula feeding are probably explained by the age difference (at enrolment) between cases and controls.

We were able to review written sources to validate history of rotavirus vaccination for $92 \%(n=197)$ of cases and $90 \%(n=249)$ of controls. There was a significant difference between cases and controls with respect to vaccination history, with $48 \%$ $(n=99)$ of cases and $91 \%(n=244)$ of controls having received at least one dose of any rotavirus vaccine $(\mathrm{P}<0.001)$. This difference was observed in all age groups. The monovalent vaccine was the most commonly used rotavirus vaccine, accounting for $92 \%(n=594)$ of all rotavirus vaccine doses $(95 \%$ $(n=176)$ for cases and $90 \%(n=418)$ for controls). Most children who had received the monovalent rotavirus vaccine had completed the full two dose schedule $(95 \%, 281 / 296)$. 


\section{Burden of rotavirus disease and clinical presentation}

Of the 46856 admissions to hospital among age eligible children in the participating hospitals during the study period, $4742(10 \%)$ were for gastroenteritis. Of the 4138 screened children admitted with gastroenteritis who provided stool samples for rapid testing, $655(16 \%)$ had positive results for rotavirus (fig $2 \Downarrow$ ). Of the 255 cases with a positive rapid test result and available result from polymerase chain reaction, $248(97 \%)$ were confirmed positive for rotavirus. The peak proportion of admissions for gastroenteritis attributable to rotavirus seemed to decrease with each rotavirus season during the study period, from $39 \%$ in March 2008 to 35\% in March 2010.

For the 215 confirmed cases included in the ATP confirmed cohort, the most commonly reported symptoms were vomiting $(89 \%, \mathrm{n}=190)$, diarrhoea $(88 \%, \mathrm{n}=189)$, behaviour change $(80 \%$, $\mathrm{n}=156)$, and fever $(80 \%, \mathrm{n}=171)$. No differences were seen in terms of presence/absence of different signs/symptoms between the children (cases) who had received both doses of the monovalent rotavirus vaccine and those who had not been vaccinated (table $3 \Downarrow$ ). In terms of disease severity, the Vesikari score could not be measured for $25 \%$ of all participants (cases and controls) because of one or several missing answers in the different elements needed to calculate the score. Among the remaining participants, $67 \%(n=40)$ of cases who had received both doses of the monovalent rotavirus vaccine were classified as "severe" according to the Vesikari score (score $\geq 11$ points) compared with $86 \%(n=69)$ of cases in the unvaccinated participants. Unvaccinated participants tended to be more dehydrated. We performed a sensitivity analysis (see appendix) with worst or best case scenario for the missing elements, which showed similar results. We found no difference in terms of treatment patterns between the two groups (table $3 \Downarrow$ ). Only one case in each group required treatment in an intensive care unit. Median duration of admission was four days (range zero to 12 days) and five days (two to eight days) in the two groups, respectively.

\section{Effectiveness of rotavirus vaccination}

For the primary analysis, we included in the logistic regression analysis only informative case-control pairs in terms of vaccination status with the monovalent rotavirus vaccine (that is, case fully vaccinated or an unvaccinated case and at least one control fully vaccinated or an unvaccinated control). Therefore we included 160 pairs (70 fully vaccinated and 90 unvaccinated cases with their 179 fully vaccinated and 19 unvaccinated matched controls). Effectiveness of two doses of the monovalent rotavirus vaccine for the prevention of admission for rotavirus gastroenteritis was $90 \%$ (95\% confidence interval $81 \%$ to $95 \%$; table $4 \Downarrow$ ). Results of the sensitivity analysis for this primary objective ranged from $76 \%$ to $93 \%$. The

effectiveness of two doses of the monovalent rotavirus vaccine was $91 \%$ (75\% to 97\%) in children aged 3-11 months, and 90\% ( $76 \%$ to $96 \%$ ) in those aged $\geq 12$ months. After adjustment for potential confounding factors in the conditional logistic regression model (table $5 \Downarrow$ ), the effectiveness of two doses of the monovalent rotavirus vaccine against admission for rotavirus gastroenteritis was $90 \%$ (79\% to $96 \%$ ) overall.

In the intention to vaccinate analysis, the effectiveness of at least one dose of any rotavirus vaccine against admission for rotavirus gastroenteritis was $91 \%(82 \%$ to $95 \%)$. The

effectiveness of at least one dose of any rotavirus vaccine was $93 \%(80 \%$ to $97 \%$ ) in children aged 3-11 months and $89 \%(75 \%$ to $95 \%$ ) in those aged 12 months or older.
In all, $56 \%(n=120)$ of cases of rotavirus gastroenteritis were classified as severe according to the Vesikari scale (score $\geq 11$ points). The effectiveness of two doses of the monovalent rotavirus vaccine against severe rotavirus gastroenteritis was $91 \%(80 \%$ to $96 \%)$. Vaccine effectiveness was $66 \%(-31 \%$ to $91 \%$ ) against rotavirus gastroenteritis of mild to moderate severity according to the Vesikari scale (score 1-10 points). The difference in vaccine effectiveness according to severity of gastroenteritis was not significant.

Of all cases of rotavirus gastroenteritis confirmed by polymerase chain reaction in the ATP confirmed cohort, $52 \%(\mathrm{n}=111)$ were G2P[4], 24\% (n=52) were G1P[8], 9\% ( $\mathrm{n}=20)$ were G4P[8], $7 \%(n=16)$ were G3P[8], and 5\% $(n=11)$ were G9P [8]. No other genotype accounted for more than one case. The effectiveness of two doses of the monovalent rotavirus vaccine was $85 \%(64 \%$ to $94 \%$ ) against G2P[4] and 95\% (78\% to 99\%) against G1P[8]. These estimates were calculated without adjustment for potential confounding factors.

Co-infection with one or more of the following intestinal viruses was observed in a quarter $(n=53)$ of cases of rotavirus gastroenteritis confirmed by polymerase chain reaction in the ATP confirmed cohort: astrovirus $(n=29,13 \%)$, adenovirus $(n=29,13 \%)$, and norovirus $(n=2,1 \%)$. The effectiveness of two doses of the monovalent rotavirus vaccine against admission for rotavirus gastroenteritis with viral co-infection was $86 \%$ (52\% to $96 \%$ ). These estimates were calculated without adjustment for potential confounding factors.

\section{Discussion}

This case-control study showed that rotavirus vaccination is effective for the prevention of admission to hospital for rotavirus gastroenteritis among young children in Belgium, despite the high prevalence of G2P[4] strains and a high rate of co-infection with other common intestinal viruses. Results of an intention to vaccinate analysis showed that at least one dose of any rotavirus vaccine can provide $91 \%$ protection against hospital admission. Estimates of vaccine effectiveness were robust, as indicated by the results of sensitivity analyses and after adjustment for potential confounding factors in the conditional logistic regression model.

With rotavirus vaccines increasingly being introduced into childhood immunisation programmes, monitoring effectiveness in real life settings is a high priority. The European Medicines Agency required evidence of field effectiveness after the introduction of the vaccine. In Latin America, the US, Europe, and Australia considerable reductions in rotavirus infections and related admissions among young children have been reported after introduction of rotavirus vaccine, ${ }^{25-36}$ with vaccination associated with a significant decline in overall deaths related to diarrhoea among children aged under 5 in Mexico. ${ }^{37}$ Such observational studies, however, were uncontrolled and potentially biased.

\section{Comparison with other studies}

Our estimates of vaccine effectiveness are comparable with the reported efficacy of both currently available rotavirus vaccines in large scale prelicensing clinical trials ${ }^{11-17}$ and similar to estimates of the effectiveness of the pentavalent rotavirus vaccine observed in case-control studies undertaken in the US. ${ }^{38}{ }^{39}$ Estimates of effectiveness of vaccine in our study were higher than have been reported in lower income settings, with the effectiveness of two doses of the monovalent rotavirus vaccine against admission for rotavirus gastroenteritis being $76 \%$ in El Salvador and Brazil ${ }^{18}{ }^{19}$ and an overall efficacy of the 
monovalent rotavirus vaccine in preventing episodes of severe rotavirus gastroenteritis of $61 \%$ in a clinical trial that was designed to simulate real world conditions of use in Malawi and South Africa. ${ }^{40}$ Research is mandatory to clearly identify the reasons for this lower potency in these challenging target countries. Micronutrient malnutrition, environmental factors, differences in the epidemiology of the virus, breast feeding at the time of vaccination, and underlying medical conditions might negatively affect the immunity of the children and performance of the monovalent vaccine. ${ }^{41} 42$

In contrast with results of case-control studies in Latin America and Central Australia that have suggested that vaccine effectiveness might decrease slightly during the second year of life, ${ }^{18} 19{ }^{43-45}$ we found no difference in effectiveness between children aged 3-11 months and those aged 12 months or older. Duration of protection is an important factor influencing the potential public health impact of rotavirus vaccines.

As observed in other case-control studies, ${ }^{18}{ }^{19}$ we found vaccination to confer greatest protection against severe disease (that is, children with scores of 11 or more on the Vesikari scale). In the present study, $44 \%$ of cases of rotavirus gastroenteritis were considered to be mild to moderate in severity according to the Vesikari scale. This was somewhat unexpected in a hospital setting. Almost all affected children required intravenous rehydration (84\%). The Vesikari scale assigns points according to the duration and severity of diarrhoea and vomiting, degree of fever, presence of dehydration, and treatment given (oral or intravenous rehydration).$^{24}$ In this study, however, we calculated Vesikari score using only data available up to the visit and not for the full duration of the episode of gastroenteritis, therefore we might have slightly underestimated severity of cases as the duration of symptoms would probably have been longer.

In line with other studies, ${ }^{19}{ }^{43}$ we found that vaccination provided effective protection against G2P[4] strains, which accounted for over half of all cases of rotavirus gastroenteritis in the present study. Cross protection is an important feature for rotavirus vaccines, considering the global strain diversity. Especially in Africa, the vaccines will need to confer protection against a wide variety of strain types, therefore it is essential to assess effectiveness in these settings. ${ }^{46} \mathrm{We}$ found evidence of co-infections with adenovirus, astrovirus, or norovirus in a quarter of all cases in this study, but these viral co-infections did not impact on vaccine effectiveness. Limited data are available on co-infection rates in rotavirus gastroenteritis, though the rate we observed in this study is higher than other recent reports. Mixed viral intestinal infections including rotavirus were reported in $13 \%$ of paediatric admission for gastroenteritis in a recent Italian study ${ }^{47}$ and in only $3.3 \%$ of children admitted for gastroenteritis in a study in northern France. ${ }^{48}$

In terms of disease burden, we found rotavirus to be responsible for about $16 \%$ of admissions for gastroenteritis among young children in Belgium. Surveillance undertaken in Belgium before introduction of rotavirus vaccine found that rotavirus gastroenteritis accounted for $58 \%$ of admission for gastroenteritis in this age group. ${ }^{6}$ The potential public health impact of rotavirus vaccination in Belgium under the current settings (that is, effectiveness of two doses of the monovalent rotavirus vaccine of $90 \%$ and $90 \%$ coverage rate) can be estimated at 4596 avoided admissions a year among children aged under 7. Our findings are in line with the reported reduction in the number of admissions attributable to rotavirus gastroenteritis in a regional Belgian hospital that was not selected for participation in this study ${ }^{29}$ and with the reduction in the number of laboratory confirmed cases of rotavirus gastroenteritis observed in Belgium after vaccine introduction. ${ }^{31}$ The proportion of admissions for rotavirus gastroenteritis in infants aged 3-5 months in our study (6.6\%) was similar to that reported in Europe before introduction of the rotavirus vaccine.$^{48}$ These findings highlight that a small but still considerable absolute number of young infants acquire rotavirus gastroenteritis, highlighting the need for a vaccine that can provide early protection against infection.

\section{Strengths and limitations of the study}

We estimated the field effectiveness of rotavirus vaccines in a post-marketing setting using a robust case-control design and investigated the potential impact of common viral intestinal co-infections on effectiveness of the vaccine. The study covered a third of all hospitals with paediatric beds in Belgium, with a common protocol, identical case definitions, and the same laboratory diagnostic methods across all participating sites. A further study strength is that nearly all vaccinated children received one type of rotavirus vaccine, which simplifies interpretation of study findings; the monovalent rotavirus vaccine accounted for $92 \%$ of all administered doses.

Although case-control studies are recognised as an effective method of assessing vaccine effectiveness in routine clinical practice, ${ }^{22}$ selection bias and other issues need to be considered in the interpretation of estimates of effectiveness.

One major concern is that ideally controls should represent the source population to which cases belong. Controls were matched by date of birth and hospital, thereby minimising the confounding bias by these factors. Although we determined that one major socioeconomic factor (number of bedrooms) was similar between cases and controls, there were significant differences in some demographic and socioeconomic variables between the two study groups, which could potentially affect effectiveness of the vaccine. We attempted to control for some of these factors in the multivariate analysis, which resulted in similar estimates of effectiveness.

The larger household size for cases compared with controls could also have resulted in increased rates of transmission of rotavirus in the households of affected children. Results of recent primary care based surveillance undertaken to estimate the burden of rotavirus gastroenteritis among children aged under 5 years in six European countries highlighted the high likelihood of transmission of rotavirus among young children within the home. ${ }^{8}$ The fact that affected children were more commonly formula fed might also have influenced the risk of developing rotavirus gastroenteritis. A recent community based study involving 30 paediatric practices in Germany, Switzerland, and Austria suggested that breast feeding might protect young infants against rotavirus gastroenteritis. ${ }^{49}$ As only $4 \%$ of controls and $2 \%$ of affected children were being breast fed, differences regarding formula feeding are probably explained by the age difference between cases and controls. Estimates of vaccine effectiveness adjusted to account for such differences between groups, however, were not significantly different to those obtained in the primary unadjusted analysis. Nevertheless, the smaller household size, the higher educational status of mothers, and the higher preschool attendance in the control group could suggest socioeconomic inequities in uptake of rotavirus vaccine (especially in a setting with partial reimbursement). These observed differences suggest that further research into possible socioeconomic inequality in access to vaccination might be warranted.

Another inherent limitation of observational studies is the possibility that the obtained history of rotavirus vaccination 
might not be correct. Rotavirus vaccination, however, is the only oral vaccine administered in Belgium, making it more easily remembered by parents. Furthermore, registration of vaccination is common practice in well baby clinics, general practitioner clinics, and other paediatric settings in Belgium, thereby reducing the chance of missing vaccination, and we reviewed written sources of vaccination history for most study participants (92\% of cases and $90 \%$ of controls). Moreover, there is an equal risk of misclassification for cases and controls and, as controls already had a high reported vaccine uptake ( $>90 \%$ ), this possible underestimate is probably minimal and will have little effect on the estimates of effectiveness as currently calculated.

It is unlikely that paediatricians might have included children with a higher chance of being vaccinated as a control. While logistical reasons prevented us from blinding the interviewers to knowledge of case and control status, identification and enrolment of the cases was not done by the same person who verified the vaccination status. Moreover, recent data show high coverage rates for vaccines implemented in the national childhood immunisation programme (for example, at least $98 \%$ of infants received three doses of the diphtheria-tetanus-pertussis vaccine) and therefore indicate that there are few barriers for vaccination in Belgium..$^{50-52}$

Finally, although a third of all paediatric departments in Belgium were included as study sites, these cases might not represent the full spectrum of severe rotavirus gastroenteritis cases in the population in Belgium.

\section{Conclusions and policy implications}

Currently available rotavirus vaccines are highly effective for the prevention of hospital admissions for rotavirus gastroenteritis among young children in Belgium under conditions of routine use. Our findings should prove useful for public health officers and policy makers to encourage implementation of rotavirus vaccine use in other similar high income countries.

We recognise the invaluable contribution of all staff involved in the conduct of this study at all the participating hospitals.

\section{RotaBel study group}

Filip Adriaens, Bert Beulens, André Bochner, Johan Colpaert, Jean De Bock, Marie-Laura Gielen, An Heyneman, Marianne Michel, Inge Matthijs, Louis Oosterlynck, Michel Pletincx, Ilse Ryckaert, Annick Sauvage, Emmi Van Damme, Ilse Vlemincx, Philippe Watillon.

Contributors: NM, PVD, MS-G, and KVH designed the study. Marcela Gavigan, Catherine Cops, Catherine Celis, Virginie Carlier, Benoit Lesage, Tine Wellens, and Sophie Vandenabeele, worked on study set up in all centres. MA, HC, JDK, A-SM, MR, LV, MV, AV and the RotaBel study group were responsible for enrolment of participants and data acquisition. $\mathrm{EH}, \mathrm{MZ}, \mathrm{JM}$, and MVR performed the laboratory analysis. TB was responsible for data acquisition, data management, training and coordination of study staff. Pascale Schrauben and Cyrille Cartier (statistical programmers) worked on the statistical analysis. NM, MS-G, $\mathrm{J}-\mathrm{YP}$, and PVD reviewed the data. TB, KVH, and PVD wrote the first draft of the manuscript. Uta Gomes and TB contributed to the publication coordination and editorial management All authors had access to the data used in this paper, contributed to the writing of the manuscript, and have seen and approved the final version.

Funding: This study was funded by GlaxoSmithKline Biologicals, which helped with study design, data collection, and analysis. GlaxoSmithKline Biologicals also funded Jennifer Coward (independent medical writer, Bollington, UK) to help with writing the paper.

Competing interests: All authors have completed the ICMJE uniform disclosure form at www.icmje.org/coi_disclosure.pdf (available on request from the corresponding author) and declare: no support from any organisation for the submitted work; no financial relationships with any organisations that might have an interest in the submitted work in the previous three years; no other relationships or activities that could appear to have influenced the submitted work.

Ethical approval: This study was approved by the local ethics committees of all participating hospitals and the ethics committee at Antwerp University Hospital. Written informed consent was obtained from the parents/guardians of all participating children before to any study procedures.

Data sharing: No additional data available.

1 Parashar UD, Hummelman EG, Bresee JS, Miller MA, Glass RI. Global illness and deaths caused by rotavirus disease in children. Emerg Infect Dis 2003;9:565-72.

2 Glass RI, Bresee J, Jiang B, Parashar U, Yee E, Gentsch J. Rotavirus and rotavirus vaccines. Adv Exp Med Biol 2006;582:45-54.

3 Cortese MM, Parashar UD; Centers for Disease Control and Prevention (CDC). Prevention of rotavirus gastroenteritis among infants and children: recommendations of the Advisory Committee on Immunization Practices (ACIP). MMWR Recomm Rep 2009;58:1-25.

4 Giaquinto C, Van Damme P; REVEAL Study Group. Age distribution of paediatric rotavirus gastroenteritis cases in Europe: the REVEAL study. Scand J Infect Dis 2010:42:142-7.

5 Soriano-Gabarró M, Mrukowicz J, Vesikari T, Verstraeten T. Burden of rotavirus disease in European Union countries. Pediatr Infect Dis J 2006;25(suppl 1):S7-11.

6 Van Damme P, Giaquinto C, Huet F, Gothefors L, Maxwell M, Van der Wielen M. Multicenter prospective study of the burden of rotavirus acute gastroenteritis in Europe, 2004-2005: the REVEAL study. J Infect Dis 2007;195(suppl 1):S4-16.

7 Forster J, Guarino A, Parez N, Moraga F, Roman E, Mory O, et al. Hospital-based surveillance to estimate the burden of rotavirus gastroenteritis among European children younger than 5 years of age. Pediatrics 2009;123:e393-400.

8 Diez-Domingo J, Baldo JM, Patrzalek M, Pazdiora P, Forster J, Cantarutti L, et al. Primary care-based surveillance to estimate the burden of rotavirus gastroenteritis among children aged less than 5 years in six European countries. Eur J Pediatr 2011;170:213-22.

9 Bilcke J, Van Damme P, De Smet F, Hanquet G, Van Ranst M, Beutels P. The health and economic burden of rotavirus disease in Belgium. Eur J Pediatrics 2008;167:1409-19.

10 World Health Organization. Rotavirus vaccines: an update. Wkly Epidemiol Rec 2009;84:533-40.

11 Ruiz-Palacios GM, Pérez-Schael I, Velázquez FR, Abate H, Breuer T, Clemens SC, et al. Safety and efficacy of an attenuated vaccine against severe rotavirus gastroenteritis. N Engl J Med 2006;354:11-22.

12 Vesikari T, Matson DO, Dennehy P, Van Damme P, Santosham M, Rodriguez Z, et al. Safety and efficacy of a pentavalent human-bovine (WC3) reassortant rotavirus vaccine. N Engl J Med 2006;354:23-33.

13 Vesikari T, Karvonen A, Prymula R, Schuster V, Tejedor JC, Cohen R, et al. Efficacy of human rotavirus vaccine against rotavirus gastroenteritis during the first 2 years of life in European infants: randomised, double-blind controlled study. Lancet 2007;370:1757-63.

14 Linhares AC, Velázquez FR, Pérez-Schael I, Saez-Llorens X, Abate H, Espinoza F, et al. Efficacy and safety of an oral live attenuated human rotavirus vaccine against rotavirus gastroenteritis during the first 2 years of life in Latin American infants: a randomised, double-blind, placebo-controlled phase III study. Lancet 2008;371:1181-9.

15 Phua KB, Lim FS, Lau YL, Nelson EA, Huang LM, Quak SH, et al. Safety and efficacy of human rotavirus vaccine during the first 2 years of life in Asian infants: randomised, double-blind controlled study. Vaccine 2009:27:5936-41.

16 Vesikari T, Itzler R, Karvonen A, Korhonen T, Van Damme P, Behre U, et al. RotaTeq, a pentavalent rotavirus vaccine: efficacy and safety among infants in Europe. Vaccine 2009;28:345-51.

17 Madhi SA, Cunliffe NA, Steele D, Witte D, Kirsten M, Louw C, et al. Effect of human rotavirus vaccine on severe diarrhea in African infants. N Engl J Med 2010;362:289-98.

18 De Palma O, Cruz L, Ramos H, de Baires A, Villatoro N, Pastor D, et al. Effectiveness of rotavirus vaccination against childhood diarrhoea in El Salvador: case-control study. BMJ 2010;341:c2825

19 Justino MC, Linhares AC, Lanzieri TM, Miranda Y, Mascarenhas JD, Abreu E, et al. Effectiveness of the monovalent G1P[8] human rotavirus vaccine against hospitalization for severe G2P[4] rotavirus gastroenteritis in Belem, Brazil. Pediatr Infect Dis J 2011;30:396-401.

20 Braeckman T, Van Herck K, Raes M, Vergison A, Sabbe M, Van Damme P. Rotavirus vaccines in Belgium: policy and impact. Pediatr Infect Dis J 2011;30(suppl 1):S21-4.

21 Bilcke J, Van Damme P, Beutels P. Cost-effectiveness of rotavirus vaccination: exploring caregiver(s) and "no medical care" disease impact in Belgium. Med Decis Making 2009;29:33-50.

22 World Health Organization. Generic protocol for monitoring impact of rotavirus vaccination on gastroenteritis disease burden and viral strains. World Health Organization, 2008.

23 Bilcke J, Beutels P, De Smet F, Hanquet G, Van Ranst M, Van Damme P. Cost-effectiveness analysis of rotavirus vaccination of Belgian infants. The Belgian Health Care Knowledge Centre (KCE), Report 54C, 2007.https://kce.fgov.be/nl/publication/report/ kosten-effectiviteitsanalyse-van-rotavirus-vaccinatie-van-zuigelingen-in-belgi\% $\mathrm{C} 3 \% \mathrm{AB}$.

24 Ruuska T, Vesikari T. Rotavirus disease in Finnish children: use of numerical scores for clinical severity of diarrhoeal episodes. Scand J Infect Dis 1990;22:259-67.

25 Tate JE, Panozzo CA, Payne DC, Patel MM, Cortese MM, Fowlkes AL, et al. Decline and change in seasonality of US rotavirus activity after the introduction of rotavirus vaccine. Pediatrics 2009;124:465-71.

26 Field EJ, Vally H, Grimwood K, Lambert SB. Pentavalent rotavirus vaccine and prevention of gastroenteritis hospitalizations in Australia. Pediatrics 2010;126:e506-12.

27 Paulke-Korinek M, Rendi-Wagner P, Kundi M, Kronik R, Kollaritsch H. Universal mass vaccination against rotavirus gastroenteritis: impact on hospitalization rates in Austrian children. Pediatr Infect Dis J 2010;29:319-23.

28 Quintanar-Solares M, Yen C, Richardson V, Esparza-Aguilar M, Parashar UD, Patel MM. Impact of rotavirus vaccination on diarrhea-related hospitalizations among children $<5$ years of age in Mexico. Pediatr Infect Dis J 2010;30(suppl 1):S11-5. 


\section{What is already known on this topic}

Rotavirus vaccines have been shown to be highly efficacious in large scale phase III prelicensing clinical trials Effectiveness of rotavirus vaccine in routine use has been reported mainly in low and middle income settings

\section{What this study adds}

Rotavirus vaccination is effective for the prevention of hospital admissions for rotavirus gastroenteritis in young children in Belgium, providing protection equivalent to that seen in clinical trial settings

Vaccine effectiveness was maintained during the second year of life

Rotavirus vaccination was highly effective, despite the high prevalence of G2P[4] strains and a high rate of co-infection with other common gastrointestinal viruses

29 Zeller M, Rahman M, Heylen E, De Coster S, De Vos S, Arijs I, et al. Rotavirus incidence and genotype distribution before and after national rotavirus vaccine introduction in Belgium. Vaccine 2010;28:7507-13

30 Buttery JP, Lambert SB, Grimwood K, Nissen MD, Field EJ, Macartney KK, et al. Reduction in rotavirus-associated acute gastroenteritis following introduction of rotavirus vaccine into Australia's National Childhood vaccine schedule. Pediatr Infect Dis J 2011;30(supp 1):S25-9.

31 Hanquet G, Ducoffre G, Vergison A, Neels P, Sabbe M, Van Damme P, et al. Impact of rotavirus vaccination on laboratory confirmed cases in Belgium. Vaccine 2011;29:4698-703.

32 Molto Y, Cortes JE, De Oliveira LH, Mike A, Solis I, Suman O, et al. Reduction of diarrhea-associated hospitalizations among children aged $<5$ years in Panama following the introduction of rotavirus vaccine. Pediatr Infect Dis J 2011;30(suppl 1):S16-20.

33 Raes M, Strens D, Vergison A, Verghote M, Standaert B. Reduction in pediatric rotavirus-related hospitalizations after universal rotavirus vaccination in Belgium. Pediatr Infect Dis J 2011;30:e120-5.

34 Tate JE, Mutuc JD, Panozzo CA, Payne DC, Cortese MM, Cortes JE, et al. Sustained decline in rotavirus detections in the United States following the introduction of rotavirus vaccine in 2006. Pediatr Infect Dis J 2011;30(suppl 1):S30-4.

35 Yen C, Armero Guardado JA, Alberto P, Rodriguez Araujo DS, Mena C, Cuellar E, et al. Decline in rotavirus hospitalizations and health care visits for childhood diarrhea following rotavirus vaccination in El Salvador. Pediatr Infect Dis J 2011;30(suppl 1):S6-10.

36 Yen C, Tate JE, Wenk JD, Harris JM 2nd, Parashar UD. Diarrhea-associated hospitalizations among US children over 2 rotavirus seasons after vaccine introduction. Pediatrics 2011;127:e9-15.

37 Richardson V, Hernandez-Pichardo J, Quintanar-Solares M, Esparza-Aguilar M, Johnson B, Gomez-Altamirano CM, et al. Effect of rotavirus vaccination on death from childhood diarrhea in Mexico. N Engl J Med 2010;362:299-305.

38 Boom JA, Tate JE, Sahni LC, Rench MA, Hull JJ, Gentsch JR, et al. Effectiveness of pentavalent rotavirus vaccine in a large urban population in the United States. Pediatrics 2010;125:e199-207.

39 Desai SN, Esposito DB, Shapiro ED, Dennehy PH, Vázquez M. Effectiveness of rotavirus vaccine in preventing hospitalization due to rotavirus gastroenteritis in young children in Connecticut, USA. Vaccine 2010;28:7501-6.

40 Madhi SA, Cunliffe NA, Steele D, Witte D, Kirsten M, Louw C, et al. Effect of human rotavirus vaccine on severe diarrhea in African infants. N Engl J Med 2010;362:289-98.

41 Patel M, Shane AL, Parashar UD, Jiang B, Gentsch JR, Glass RI. Oral rotavirus vaccines: how well will they work where they are needed most? J Infect Dis 2009;200 (suppl 1):S39-48.

42 Chan J, Nirwati H, Triasih R, Bogdanovic-Sakran N, Soenarto Y, Hakimi M, et al. Maternal antibodies to rotavirus: could they interfere with live rotavirus vaccines in developing countries? Vaccine 2011;29:1242-7.
43 Correia JB, Patel MM, Nakagomi O, Montenegro FM, Germano EM, Correia NB, et al. Effectiveness of monovalent rotavirus vaccine (Rotarix ${ }^{T M}$ ) against severe diarrhea caused by serotypically unrelated G2P[4] strains in Brazil. $J$ Infect Dis 2010;201:363-9.

44 Patel M, Pedreira C, De Oliveira LH, Tate J, Orozco M, Mercado J, et al. Association between pentavalent rotavirus vaccine and severe rotavirus diarrhea among children in Nicaragua. JAMA 2009;301:2243-51.

45 Snelling TL, Andrews RM, Kirkwood CD, Culvenor S, Carapetis JR. Case-control evaluation of the effectiveness of the G1P[8] human rotavirus vaccine during an outbreak of rotavirus G2P[4] infection in Central Australia. Clin Infect Dis 2011;52:191-9.

46 Todd S, Page NA, Duncan Steele A, Peenze I, Cunliffe NA. Rotavirus strain types circulating in Africa: review of studies published during 1997-2006. J Infect Dis 2010;202(suppl):S34-42

47 Rimoldi SG, Stefani F, Pagani C, Chenal LL, Zanchetta N, Di Bartolo I, et al. Epidemiological and clinical characteristics of pediatric gastroenteritis associated with new viral agents. Arch Virol 2011;156:1583-9.

48 Tran A, Talmud D, Lejeune B, Jovenin N, Renois F, Payan C, et al. Prevalence of rotavirus, adenovirus, norovirus, and astrovirus infections and coinfections among hospitalized children in northern France. J Clin Microbiol 2010;48:1943-6.

49 Plenge-Bönig A, Soto-Ramírez N, Karmaus W, Petersen G, Davis S, Forster J. Breastfeeding protects against acute gastroenteritis due to rotavirus in infants. Eur $J$ Pediatr 2010;169:1471-6.

50 Hoppenbrouwers K, Vandermeulen C, Roelants M, Boonen M, Van Damme P, Theeten $\mathrm{H}$, et al. Vaccination coverage survey in infants and adolescents in Flanders in 2008. 2009. www.zorg-en-gezondheid.be/Cijfers/Ziekten/Infectieziekten-en-vaccinatie/ Vaccinatiegraadstudies/.

51 Boonen M, Theeten H, Vandermeulen C, Roelants M, Depoorter A-M, Van Damme P, et al. Vaccinatiegraad bij jonge kinderen en adolescenten in Vlaanderen in 2008. Vlaams Infectieziektebulletin 2009;68:9-14

52 Robert E, Swennen B. Enquête de couverture vaccinale des enfants de 18 à 24 mois en communauté française (Bruxelles excepté). PROVAC, School of Public Health ULB, 2009.

\section{Accepted: 13 June 2012}

\section{Cite this as: BMJ 2012:345:e4752}

This is an open-access article distributed under the terms of the Creative Commons Attribution Non-commercial License, which permits use, distribution, and reproduction in any medium, provided the original work is properly cited, the use is non commercial and is otherwise in compliance with the license. See: http://creativecommons.org/licenses/by$\mathrm{nc} / 2.0 /$ and http://creativecommons.org/licenses/by-nc/2.0/legalcode. 


\section{Tables}

Table 1| Demographic characteristics in all children with rotavirus confirmed by polymerase chain reaction and having at least one valid control (according to protocol, confirmed cohort) and matched controls. Figures are numbers (percentage) unless stated otherwise

\section{Characteristic}

Children with rotavirus $(n=215)$

Controls $(n=276)$

Age at onset ${ }^{*}$ (months):

\begin{tabular}{lcc}
\hline Mean & 13.0 & 12.6 \\
\hline Median (range) & $11(2-31)$ & $12(2-31)$ \\
\hline Age at onset ${ }^{*}$ (months): & & $1(0.4)$ \\
\hline $0-2 \dagger$ & $1(0.5)$ & $20(7)$ \\
\hline $3-5$ & $16(7)$ & $118(43)$ \\
\hline $6-11$ & $87(41)$ & $117(42)$ \\
\hline $12-23$ & $93(43)$ & $20(7)$ \\
\hline$\geq 24$ & $18(8)$ & $148(54)$ \\
\hline Boys & $109(51)$ &
\end{tabular}

*For controls, age is computed at date of onset of disease in matched case.

†Age at onset could be lower than 3 months given that inclusion criteria were "at least 14 weeks of age at time of hospital admission" and "onset of severe gastroenteritis $\leq 14$ days before admission." Two children $<3$ months were exactly 89 days old (for case) and 81 days old (for control). 
Table 2/ Clinical and socioeconomic characteristics in all children with rotavirus confirmed by polymerase chain reaction and having at least one valid control (according to protocol, confirmed cohort) and matched controls. Figures are numbers (percentages) unless otherwise stated

\begin{tabular}{|c|c|c|c|}
\hline Characteristic & Children with rotavirus $(n=215)$ & Controls $(n=276)$ & $P$ value \\
\hline Previous admission to hospital for gastroenteritis & $25(12)$ & $24(9)$ & 0.29 \\
\hline \multicolumn{4}{|l|}{ Medical history: } \\
\hline Premature birth & $14(7)$ & $23(9)$ & 0.60 \\
\hline Cardiac disease & $4(2)$ & $6(2)$ & 1.00 \\
\hline Pulmonary disease & $35(17)$ & $49(18)$ & 0.81 \\
\hline Gastrointestinal disease & $21(10)$ & $38(14)$ & 0.21 \\
\hline Other & $36(17)$ & $57(21)$ & 0.35 \\
\hline \multicolumn{4}{|l|}{ Current feeding practice*: } \\
\hline Breast fed & $4(2)$ & $12(4)$ & 0.20 \\
\hline Formula fed & $112(52)$ & $105(38)$ & 0.003 \\
\hline Solid food & $192(89)$ & $250(901)$ & 0.65 \\
\hline \multicolumn{4}{|l|}{ Highest level of maternal education†: } \\
\hline None & $1(0.5)$ & $0(0.0)$ & 0.0039 \\
\hline Primary school & $10(5)$ & $2(0.8)$ & \\
\hline Secondary school & $96(46)$ & $101(38)$ & \\
\hline Higher education ( $\leq 3$ years) & $67(32)$ & $113(43)$ & \\
\hline Higher education (>3 years) & $33(16)$ & $48(18)$ & \\
\hline Mean (SD) No of bedrooms in home & $3.0(1.3)$ & $2.9(0.9)$ & 0.29 \\
\hline Mean (SD) No of people spending $>3$ nights/week in home & $4.2(2)$ & $3.6(0.8)$ & $<0.001$ \\
\hline Daycare attendance & $125(60)$ & $116(62)$ & 0.64 \\
\hline Preschool attendance & $1(0.5)$ & $21(8)$ & $<0.001$ \\
\hline
\end{tabular}


Table 3/ Clinical characteristics and management of rotavirus gastroenteritis in children who had received both doses of monovalent rotavirus vaccines (vaccinated cases) and those who had not received any rotavirus vaccination (unvaccinated cases) in children with rotavirus confirmed by polymerase chain reaction and having at least one valid control (according to protocol, confirmed cohort). Figures numbers (percentages) unless otherwise stated

\begin{tabular}{|c|c|c|c|c|}
\hline \multirow[b]{2}{*}{ Characteristic } & \multicolumn{2}{|c|}{ Fully vaccinated ${ }^{*}(n=83)$} & \multicolumn{2}{|c|}{ Unvaccinated $+(n=108)$} \\
\hline & No $(\%$ or SD) & Missing & No $(\%$ or SD) & Missing \\
\hline \multicolumn{5}{|l|}{ Symptoms: } \\
\hline Vomiting & $71(86)$ & 0 & $97(91)$ & 1 \\
\hline Diarrhoea & $75(90)$ & 0 & $95(89)$ & 1 \\
\hline Behaviour change & $59(80)$ & 9 & $80(80)$ & 8 \\
\hline Fever & $65(78)$ & 0 & $85(80)$ & 2 \\
\hline \multicolumn{5}{|l|}{ Severity (Vesikari scale) ${ }^{24}$ : } \\
\hline Mild/moderate (1-10) & $20(33)$ & 23 & $11(14)$ & 28 \\
\hline Severe $(\geq 11)$ & $40(67)$ & & $69(86)$ & \\
\hline \multicolumn{5}{|l|}{ Treatment received: } \\
\hline Oral rehydration & $65(79)$ & 1 & $76(72)$ & 2 \\
\hline Intravenous rehydration & $70(84)$ & 0 & $89(84)$ & 2 \\
\hline Intensive care & $1(1.2)$ & 1 & $1(0.9)$ & 2 \\
\hline \multicolumn{5}{|c|}{ Duration of admission (days): } \\
\hline Median (range) & $4(1-12)$ & 0 & $5(2-8)$ & 0 \\
\hline Mean (SD) & $4.6(1.9)$ & & $4.5(1.4)$ & \\
\hline
\end{tabular}

*Children who had received both doses of monovalent rotavirus vaccine (information regarding two children partially vaccinated with monovalent rotavirus vaccine, three children vaccinated with three doses of pentavalent rotavirus vaccine $(n=3)$, those in category "other" ( $n=11)$, and those in category "unknown" ( $n=8)$ is not shown).

†Children who had not received any rotavirus vaccination. 
Table 4| Effectiveness of human rotavirus vaccine against admission to hospital for rotavirus gastroenteritis (Belgium, February 2008-June 2010) in all children with rotavirus confirmed by polymerase chain reaction and having at least one valid control (according to protocol, confirmed cohort) and matched controls. Estimates of effectiveness are not adjusted for potential confounding variables

\begin{tabular}{|c|c|c|c|c|c|}
\hline & \multicolumn{2}{|c|}{ No/total receiving vaccine } & \multirow{2}{*}{$\begin{array}{l}\text { Vaccine effectiveness (\% } \\
(95 \% \mathrm{Cl}))\end{array}$} & \multicolumn{2}{|c|}{ Sensitivity $(\%)^{\star}$} \\
\hline & Children with rotavirus & Matched controls & & - & + \\
\hline \multicolumn{6}{|c|}{ Received two doses of monovalent vaccine } \\
\hline Overall & $70 / 160$ & $179 / 198$ & 90 (81 to 95$)$ & 76 & 93 \\
\hline \multicolumn{6}{|l|}{ Age (months): } \\
\hline $3-11$ & $30 / 66$ & $79 / 84$ & 91 (75 to 97 ) & 69 & 94 \\
\hline$\geq 12$ & $40 / 94$ & $100 / 114$ & 90 (76 to 96$)$ & 82 & 91 \\
\hline \multicolumn{6}{|l|}{ Co-infection status: } \\
\hline Not co-infected & $50 / 121$ & $134 / 148$ & 91 (81 to 96$)$ & 78 & 93 \\
\hline Co-infected & $20 / 39$ & $45 / 50$ & $86(52$ to 96$)$ & 69 & 90 \\
\hline \multicolumn{6}{|l|}{ Genotypet: } \\
\hline G1P[8] & $11 / 41$ & $49 / 53$ & 95 (78 to 99$)$ & 92 & 95 \\
\hline G2P[4] & $46 / 80$ & $93 / 103$ & 85 (64 to 94$)$ & 54 & 89 \\
\hline \multicolumn{6}{|c|}{ Severity (Vesikari scale)‡: } \\
\hline Mild/moderate $(1-10)$ & $16 / 26$ & $28 / 34$ & $66(-31$ to 91$)$ & 8 & 73 \\
\hline Severe $(\geq 11)$ & $38 / 96$ & $110 / 121$ & 91 (80 to 96$)$ & 88 & 93 \\
\hline \multicolumn{6}{|c|}{ Received at least one dose of any rotavirus vaccine } \\
\hline Overall & $81 / 179$ & $208 / 228$ & 91 (82 to 95$)$ & 78 & 93 \\
\hline \multicolumn{6}{|l|}{ By age (months): } \\
\hline 3-11 & $36 / 77$ & $99 / 104$ & $93(80$ to 97$)$ & 75 & 95 \\
\hline$\geq 12$ & $45 / 102$ & $109 / 124$ & 89 (75 to 95$)$ & 81 & 90 \\
\hline
\end{tabular}

*Sensitivity-: cases and controls with other or unknown vaccination status are assumed, respectively, to be vaccinated and unvaccinated; sensitivity + : cases and controls with other or unknown vaccination status are assumed, respectively, to be unvaccinated and vaccinated

†Only effectiveness results for pairs in which case had either genotype G1P[8] or G2P[4] could be estimated, as for other genotypes numbers were too small. ¥Severity missing for 38 cases (matched to 43 controls). 
Table 5| Estimated coefficients of final fitted logistic regression model for effectiveness of two doses of monovalent rotavirus vaccine against admission to hospital for rotavirus gastroenteritis (Belgium, February 2008-June 2010) in all children with rotavirus confirmed by polymerase chain reaction and having at least one valid control (according to protocol, confirmed cohort) and matched controls

\begin{tabular}{|c|c|c|c|c|}
\hline Parameter & $\begin{array}{l}\text { No }(\%) \text { of children with } \\
\text { rotavirus }(n=145)^{\star}\end{array}$ & No $(\%)$ of controls $(n=182)$ & $P$ value & Adjusted odds ratio $(95 \% \mathrm{Cl})$ \\
\hline Not vaccinated (reference) & $79(54)$ & $17(9)$ & - & - \\
\hline $\begin{array}{l}\text { Two doses of monovalent rotavirus } \\
\text { vaccine }\end{array}$ & $66(46)$ & $165(91)$ & $<0.001$ & $0.10(0.05$ to 0.21$)$ \\
\hline Gastrointestinal diseases (yes $v$ no $\dagger$ ) & - & - & 0.188 & 0.51 (0.19 to 1.39$)$ \\
\hline Pulmonary disease (yes $v$ not) & - & - & 0.112 & $0.45(0.16$ to 1.21$)$ \\
\hline Household sizeł & - & - & 0.048 & $1.40(1.00$ to 1.94$)$ \\
\hline
\end{tabular}

*Because logistic regression model requires complete data, 15 ( 15 cases and their 16 matched controls) out of 160 pairs available for primary analysis were excluded because of missing information for at least one of variables included in model.

†Reference category

$\ddagger$ Number of people sleeping in house for more than three nights a week. 


\section{Figures}

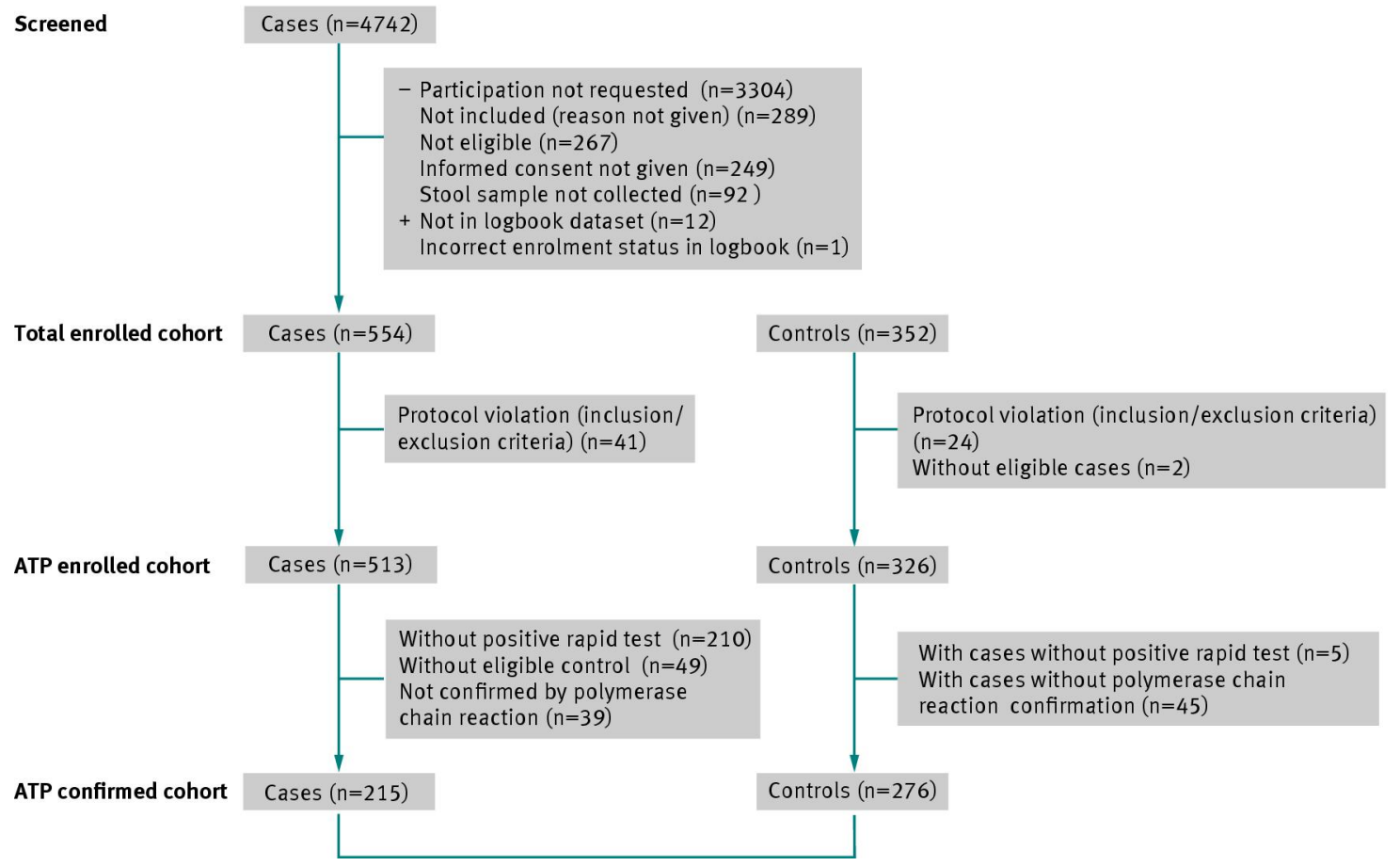

Fig 1 Summary of enrolment by cohort. Screened cohort=all children aged $\geq 14$ weeks and born after 1 October 2006 admitted with gastroenteritis. Total enrolled cohort=all children (cases and controls) for whom informed consent was obtained. ATP enrolled cohort=all valid enrolled cases and controls. ATP confirmed cohort=all valid cases confirmed by polymerase chain reaction with at least one valid control and their matched controls (used for analyses of vaccine effectiveness); (154 cases have 1 matched control, 61 cases have 2 matched controls)

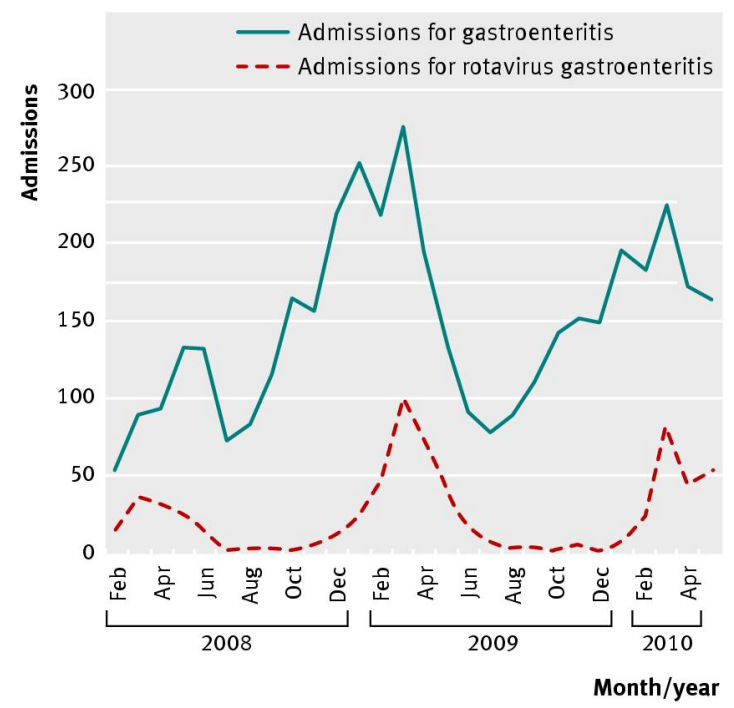

Fig 2 Number of admissions attributable to gastroenteritis and rotavirus gastroenteritis (in according to protocol (ATP) enrolled cohort) 